\title{
Quedas em idosos com dor crônica: prevalência e fatores associados *
}

\section{Falls in chronic pain elderly patients: prevalence and associated factors}

Heloísa Mussato Fernandes da Cruz¹, Cibele Andrucioli de Mattos Pimenta², Mara Solange Gomes Dellarozza ${ }^{3}$

Patricia Emilia Braga4, Maria Lúcia Lebrão5 ${ }^{5}$ Yeda Aparecida Oliveira Duarte ${ }^{6}$

* Recebido da Escola de Enfermagem da Universidade de São Paulo (USP). São Paulo, SP.

- Com financiamento da CNPq Edital universal de 2009.

\section{RESUMO}

JUSTIFICATIVA E OBJETIVOS: A prevalência de queda e dor crônica é elevada em idosos com importantes consequências para a qualidade de vida. Verificar associação entre estes dois agravos pode ajudar a prevenir incapacidades e sofrimentos. O objetivo deste estudo foi identificar a prevalência de quedas entre idosos com dor há mais de um ano e avaliar a associação entre a ocorrência de quedas e a localização, intensidade e frequência da dor. MÉTODO: Estudo transversal utilizando dados do projeto Saúde, Bem Estar e Envelhecimento. As variáveis dependentes foram: ocorrência e número de queda nos últimos 12 meses. As variáveis independentes foram: a localização, intensidade e frequência da dor que "mais

1. Graduanda da Escola de Enfermagem da Universidade de São Paulo. São Paulo, SP, Brasil.

2. Professora Titular do Departamento de Enfermagem Médico-Cirúrgica da Escola de Enfermagem da Universidade de São Paulo. São Paulo, SP, Brasil.

3. Aluna de Doutorado do Programa de Pós-Graduação em Enfermagem na Saúde do Adulto da Escola de Enfermagem da Universidade de São Paulo. São Paulo, SP, Brasil.

4. Estatística, Aluna de Pós-Doutorado do Programa de Pós-Graduação em Enfermagem Saúde do Adulto da Universidade de São Paulo. São Paulo, SP, Brasil.

5. Docente do Departamento Epidemiologia da Universidade de São Paulo. São Paulo, SP, Brasil.

6. Docente de Enfermagem da Universidade de São Paulo. São Paulo, SP, Brasil.

Endereço para correspondência:

Mara Solange Gomes Dellaroza

Rua Borba Gato, 1078/904

86010- 630 Londrina, PR.

Fones: (043) 3371-2309 - (043) 9998-6468

E-mail: dellaroza@uel.br incomoda" com duração de mais de um ano. Para verificação de associação, realizou-se o teste de associação de Rao Scott, indicado para amostra populacional.

RESULTADOS: Foram analisados 213 idosos acima de 60 anos com dor há mais de um ano e que responderam sobre ocorrência de quedas no último ano. A sua prevalência foi $56,29 \%$. Resultados apontaram que idosos com dor há mais de um ano, intensa ou moderada, relataram maior número de quedas do que os idosos com dores leves. Não foram encontradas associações entre local e frequência dos episódios de dor e relato de quedas no último ano.

CONCLUSÃO: Melhor controle da dor pode diminuir risco de quedas em idosos com dores crônicas, estes dois agravos precisam ser adequadamente avaliados e controlados pelos profissionais de saúde.

Descritores: Acidentes por quedas, Assistência a idosos, Dor, Medição da dor.

\section{SUMMARY}

BACKGROUND AND OBJECTIVES: The prevalence of falls and chronic pain is high among the elderly with major consequences for quality of life. Checking the association between these two problems may help preventing incapacities and distress. This study aimed at identifying the prevalence of falls among the elderly with pain lasting more than one year and at evaluating the association of falls and pain location, intensity and frequency.

METHOD: This was a transversal study using data from the Health, Wellbeing and Ageing project. Dependent variables were: occurrence and number of falls in the last 12 months. Independent variables were: location, intensity and frequency of the "most uncomfortable" pain 
lasting more than one year. Rao Scott test, indicated for population sampling, was used to check the association. RESULTS: We evaluated 213 elderly people above 60 years of age with pain lasting more than one year and who answered about falls in the last year. Its prevalence was $56.29 \%$. Results have shown that elderly people with severe or moderate pain lasting more than one year have reported a higher number of falls as compared to elderly people with mild pain. No association was found between site and frequency of pain episodes and falls in the last year.

CONCLUSION: Better pain control may decrease the risk of falls in the elderly population with chronic pain; these two problems have to be adequately evaluated and controlled by health professionals.

Keywords: Accidents with falls, Assistance to the elderly, Pain, Pain measurement.

\section{INTRODUÇÃO}

O aumento do número de idosos no Brasil é fato conhecido e sabe-se que estes indivíduos sofrem alterações sensitivas, que aumentam o risco para quedas. As quedas estão entre as principais causas de incapacidades e dependência em pessoas acima de 60 anos $^{1-4}$. Os idosos, frequentemente, têm pouca consciência dos fatores de risco para quedas a que estão sujeitos e deixam de relatar estes aspectos aos profissionais de saúde. Estes fatores podem ser intrínsecos, relacionados à comorbidades e extrínsecos, relacionados a medicamentos e meio ambiente. Para monitorar o risco de quedas deve-se realizar avaliação ampla e em intervalos curtos sobre as condições de saúde e capacidade física dos idosos ${ }^{5}$.

Entre as múltiplas causas de queda, a dor pode ser uma delas, e seu controle é uma forma de prevenção. Nos idosos a dor crônica é, geralmente, relacionada a doenças degenerativas do aparelho locomotor, doenças neuropáticas e oncológicas. Estima-se que entre $80 \% \mathrm{e}$ $85 \%$ dos indivíduos com mais de 65 anos apresentam, pelo menos, um problema significativo de saúde que os predispõem a apresentar dor e aproximadamente $50 \%$ a $60 \%$ dos pacientes tornam-se parcial ou totalmente incapacitados por ela, de modo transitório ou permanente . $^{6}$

Estudo com 245 idosos brasileiros vinculados a serviço de saúde encontrou dor crônica relatada por 166 idosos $(67,7 \%)$, as regiões mais acometidas foram membros inferiores, referidos por $66(46,8 \%)$ mulheres e por 37 $(35,6 \%)$ homens, e a coluna lombar, referida por 40 $(28,4 \%)$ mulheres e por $28(26,9 \%)$ homens. O uso de medicamentos em decorrência da dor foi referido por $120(48,9 \%)$ dos idosos 7 .
O conceito de dor da Associação Internacional para o Estudo da Dor (IASP) de 1986, que será observado neste estudo, refere-se à dor como uma experiência sensorial e emocional desagradável que é descrita em termos de lesões teciduais, reais ou potenciais. A dor é sempre subjetiva e cada individuo aprende e utiliza este termo a partir de suas experiências traumáticas ${ }^{8}$. A IASP define dor crônica como aquela com duração maior de 3 meses e, para fins de pesquisa, aquela com duração superior a 6 meses. Nota-se que é um conceito centrado no tempo. Dentro desse conceito podem estar incluídas pessoas com dores crônicas completamente diferentes, em termos de incapacidade e sofrimento. Identificar os prejuízos associados à dor crônica contribuirá para a organização de propostas de prevenção e para a diminuição desses prejuízos.

Visando analisar a literatura sobre queda em idosos com dor, foi realizada revisão bibliográfica no MedLine, com os descritores Pain (Medical Condition) and Falls or Disability (Outcome) e limitado para idade: $>65$ anos. Foram encontrados 61 resultados, e desses, 47 foram excluídos por não apresentarem relação com o estudo, quatro por não ter sido possível obter os textos na íntegra. Apenas nove estudos associavam a ocorrência de dor e quedas em idosos ${ }^{1-4,9-13}$.

Entre os estudos nove foram realizados com idosos da comunidade, seis foram realizados com idades a partir de 65 $\operatorname{anos}^{2,3,4,10,12,13}$. Seis observaram associação entre dor e que$\mathrm{da}^{1,2,9,10,11,13}$. Somente três estudos encontraram associação entre a intensidade da dor e a ocorrência de quedas ${ }^{1,2,13}$. Um estudo de coorte envolvendo mulheres $(n=1002)$ de 65 anos ou mais, que apresentavam deficiência leve em domínios de mobilidade, autocuidado básico e atividades mais sofisticadas como: realizar telefonemas, trabalhos domésticos e preparar refeições. No estudo 39\% das mulheres referiram ao menos um episódio de queda no período do estudo. A avaliação de desempenho dor segundo os instrumentos validados demonstrou uma determinação tendenciosa durante a avaliação de dor para predisposição a quedas durante o período de acompanhamento ${ }^{4}$.

Em estudo retrospectivo com 106 idosos com dor no joelho de duração de mais de 6 meses e com diagnóstico de osteoartrite, encontrou-se que $45 \%$ da amostra teve ao menos uma queda no último ano e $77 \%$ relataram episódios de "quase queda" no mesmo período. A "quase queda" é definida como deslizamento da perna de apoio, perda de equilíbrio no qual a pessoa consegue restabelecer com apoio em superfície ${ }^{11}$. Estudo comprovou que a dor é um preditor para o decréscimo no balanço e mobilidade em idosos, o que pode aumentar o risco de queda, ao avaliar 55 idosos com idade entre 65 e 95 anos dos quais $75 \%$ relataram dor, sendo 
o principal problema dor lombar ${ }^{13}$.

Estudo realizado no México com 12.459 pessoas com mais de 50 anos encontrou prevalência de dor em 41,5\% (IC 95\% 40,6-42,3). As quedas foram encontradas em $43,9 \%$ em pessoas de 50-64 anos e 51,8\% em idosos acima de 65 anos (IC 95\%1,31-1,72) sendo maior a frequência de quedas nas duas faixas etárias entre os idosos com dor $(\mathrm{p}=0,0001)$. Após análise multivariada o risco de queda entre os indivíduos com dor foi de 1,62 para a idade de 50 a 64 anos e 1,52 para mais de 65 anos controlado para sexo e escolaridade ${ }^{10}$.

A associação entre dor e queda não é amplamente investigada na literatura. Nas pesquisas que analisaram queda e dor em idosos, as características da dor como local e intensidade, entre outras, foram pouco exploradas. Assim, é importante a realização de outros estudos para verificar quais características da dor mais interferem na ocorrência de quedas. Compreender melhor esta associação permitirá implementar ações de controle da dor que podem prevenir a ocorrência de quedas.

Os objetivos do presente estudo são: identificar a prevalência de quedas entre idosos com dor há mais de um ano e verificar a associação entre ocorrência de quedas e a localização, intensidade e frequência da dor que mais incomoda, em idosos com dores crônicas.

\section{MÉTODO}

Após aprovação pelo Comitê de Ética da Faculdade de Saúde Pública da Universidade de São Paulo, realizou-se este estudo, em que se obteve autorização dos coordenadores do Projeto Saúde, Bem Estar e Envelhecimento (SABE) para utilização dos dados.

Estudo transversal utilizando dados oriundos do projeto SABE, que é um estudo longitudinal multicêntrico financiado pela Organização Pan-Americana de Saúde e
FAPESP, cujo objetivo é analisar o impacto do envelhecimento populacional em países da América. No que tange ao Brasil, a amostra populacional constituiu-se de idosos residentes em São Paulo, buscou garantir a representação da população segundo estratos definidos por sexo e idade. Os idosos foram localizados por sorteio de domicílios, distribuídos em 72 setores censitários disponíveis no Departamento de Epidemiologia da Faculdade de Saúde Pública. O SABE é um estudo de coorte no qual foram feitas duas coletas, a primeira no ano 2000 e outra em 2006. Os dados foram coletados por entrevista, com preenchimento dos questionários durante a visita domiciliar e atualizados por contato telefônico. Para o presente estudo foi utilizado dados da coleta de 2006, na qual foram acrescentadas questões sobre dor crônica em geral.

No presente estudo foram analisados entre os idosos acima de 60 anos $(\mathrm{n}=1271)$, os com dor há mais de um ano $(\mathrm{n}=340)$, e destes 213 que responderam sobre a ocorrência de quedas no último ano. Como os idosos foram avaliados quanto à presença de várias dores, no presente estudo, optou-se por utilizar nas análises, a dor que mais incomoda e com duração a mais de um ano.

\section{Variáveis dependentes}

As variáveis dependentes foram: ocorrência de queda e número de quedas nos últimos 12 meses. Divididas em duas categorias: "com queda" os idosos que afirmaram sim a pergunta: Teve alguma queda nos últimos 12 meses? E "sem queda ou sem resposta" incluídos os idosos que afirmaram não terem tido quedas ou os que não responderam a pergunta.

\section{Variáveis independentes}

Para a dor que "mais incomoda" com duração de mais de um ano, os idosos forneceram informações sobre a localização, intensidade e frequência, variáveis independentes deste estudo (Quadro 1).

Quadro 1 - Categorias das variáveis independentes referentes às características da dor que mais incomoda há mais de um ano

\begin{tabular}{lll}
\hline Características da Dor & Descritores & $\begin{array}{l}\text { Categorias Utilizadas } \\
\text { nas Análises }\end{array}$ \\
\hline $\begin{array}{l}\text { Local da dor que mais } \\
\text { incomoda }\end{array}$ & $\begin{array}{l}\text { Cabeça/ face e boca, pescoço, ombros e braços, peito, } \\
\text { costas (acima da cintura), costas (na cintura e abaixo - } \\
\text { região lombar, abdômen, pernas, pelve, região anal e genital; }\end{array}$ & $\begin{array}{l}\text { Membros Inferiores, Lombar, } \\
\text { somente em outras partes }\end{array}$ \\
\hline $\begin{array}{l}\text { Intensidade da dor que } \\
\text { mais incomoda há mais } \\
\text { de um ano }\end{array}$ & $\begin{array}{l}\text { Muito forte/ muito intensa, forte/ intensa } \\
\text { Fraca }\end{array}$ & $\begin{array}{l}\text { Intensa } \\
\text { Moderada }\end{array}$ \\
\hline $\begin{array}{l}\text { Frequência da dor } \\
\text { que mais incomoda }\end{array}$ & $\begin{array}{l}\text { Quase todos os dias } \\
\text { Uma ou duas vezes por semana }\end{array}$ & Fma vez a cada 15 dias \\
& Uma vez por mês & $\begin{array}{l}\text { Quase todos os dias } \\
\text { Uma ou duas vezes por semana } \\
\text { Uma vez a cada 15 dias }\end{array}$ \\
\hline
\end{tabular}


Os dados foram inseridos e analisados no banco de dados do programa estatístico STATA 9.0 (StataCorp LP, College Station, Texas, USA). Estão apresentados em porcentagem, por se tratar de uma amostra populacional. Para verificação de associação entre quedas e as características da dor, realizaram-se testes de associação de RaoScott, indicado para amostra populacional.

\section{RESULTADOS}

A tabela 1 apresenta as características sócio-demográficas da população estudada. A maioria era do sexo feminino (73,95\%), com idade entre 65 e 74 anos $(71,8 \%)$, não trabalhava no momento da entrevista $(70,44 \%)$ e vivia com companheiro $(84,68 \%)$. Notou-se que $41,42 \%$ possuíam renda familiar de 1 a 3 salários mínimos.

Tabela 1 - Distribuição dos idosos com dor há mais de um ano segundo características sócio-demográficas - São Paulo 2006.

\begin{tabular}{ll}
\hline Variáveis & $\%$ \\
\hline Sexo & \\
Feminino & 73,95 \\
Masculino & 26,05 \\
Idade (anos) & \\
65 a 74 & 71,80 \\
$\geq 75$ & 28,20 \\
Média (ep) & $70,8(0,7)$ \\
Situação ocupacional $(\mathrm{n}=200)$ & \\
Trabalha & 29,56 \\
Não trabalha & 70,44 \\
Escolaridade (anos) & \\
Analfabeto & 17,71 \\
1 a 3 & 29,24 \\
4 a 7 & 36,37 \\
$\geq 8$ & 16,68 \\
Média (ep) & $4,1(0,4)$ \\
Vive sozinho & \\
Sim & 15,32 \\
Não & 84,68 \\
Renda familiar (SM) (n=204) & \\
< 1 $1 \vdash 3$ & 31,44 \\
$3 \vdash 5$ & 41,42 \\
$\geq 5$ & 13,44 \\
Média (ep) & 13,70 \\
\hline
\end{tabular}

A prevalência de queda autorreferida pelos idosos nos últimos 12 meses foi de 56,29\% [IC95\%: 47,33 - 65,24]. Dentre os que tiveram queda nesse período, $58,89 \%$ re- feriram um episódio, 13,46\% dois episódios e 27,64\% três episódios.

Na tabela 2 observa-se a associação entre ocorrência de queda nos últimos 12 meses e a intensidade da dor ( $\mathrm{p}=$ $0,0454)$. Não se observou associação entre queda a localização e a frequência de dor.

Tabela 2 - Associação entre queda e as variáveis: local, intensidade e frequência da dor há mais de um ano e que mais incomoda - São Paulo 2006.

Ocorrência de Quedas

\begin{tabular}{lrrc} 
Variáveis & Sim & Não & Valor de p \\
\hline Local da dor & & & 0,5370 \\
Membros inferiores & 48,05 & 51,95 & \\
Região lombar & 58,45 & 41,55 & \\
Outros locais & 58,03 & 41,97 & \\
Intensidade da dor & & & 0,0454 \\
Intensa & 63,43 & 36,57 & \\
Moderada & 52,27 & 47,73 & \\
Fraca & 24,10 & 75,90 & \\
Frequência da dor (n = 263) & & 0,6457 \\
Todos os dias & 50,82 & 49,18 & \\
1 ou 2 vezes & 57,91 & 42,09 & \\
por semana & & & \\
1 vez a cada 15 dias & 61,15 & 38,85 & \\
1 vez por mês & 63,10 & 36,90 & \\
\hline
\end{tabular}

Referiram queda $63,43 \%$, dos idosos com dor intensa, e $52,27 \%$ daqueles com dor moderada $(\mathrm{p}=0,0454)$.

Quanto ao local 58,45\% dos idosos com dor lombar e $58,03 \%$ dos com dor em outras partes do corpo relataram queda no último ano. Apesar das porcentagens serem maiores do que os que não relataram queda, a diferença não foi estatisticamente significante.

Não houve associação entre queda e frequência da dor que mais incomoda $(p=0,6457)$. A frequência mais referida foi todos os dias, tanto pelos idosos que não apresentaram quedas quanto para aqueles que apresentaram (54,65\% e 44,94\%, respectivamente).

\section{DISCUSSÃO}

O primeiro objetivo deste estudo foi verificar a prevalência de quedas entre idosos com dor que mais incomoda com duração maior que um ano e, a prevalência observada foi 56,29\%. 
Em estudo brasileiro do tipo longitudinal com 1667 idosos acima de 65 anos. Cerca de $31 \%$ dos idosos referiram queda no ano anterior ao primeiro inquérito realizado, sendo que $11 \%$ apresentaram dois ou mais episódios. No segundo inquérito realizado, 29,1\% referiram queda no ano anterior e $13,9 \%$ referiram queda nos dois inquéritos realizados ${ }^{14}$.

Em pesquisa do tipo transversal com 240 indivíduos com idade acima de 50 anos que avaliou dor musculoesquelética na parte inferior do corpo e história de quedas. Os participantes foram divididos em dois grupos: dor com duração inferior a um ano $(37,5 \%)$ e dor com duração superior a um ano $(62,5 \%)$. Desses, $70,4 \%$ relataram mais de um local de dor, sendo os mais referidos: lombar $11,2 \%(n=27)$ e joelho $27 \%(n=65)$. Entre os participantes de 65 a 75 anos, 25,3\% apresentaram ocorrência de quedas e 13,9\% referiram 1 ou 2 episódios de queda no último ano ${ }^{11}$.

Estudo em idosos com dor no joelho há mais 6 meses e com diagnóstico de osteoartrite encontrou que $45 \%$ teve ao menos uma queda no último ano e $77 \%$ relataram episódios de "quase queda" no mesmo período ${ }^{10}$. A prevalência de quedas é maior do que a encontrada em outros estudos, podendo este achado estar relacionado à associação com osteoartrite. A ocorrência de quase queda nesse estudo foi elevada levando-nos a refletir que o número de estudos que analisam esse episódio é baixo, frente ao grande número de idosos com dificuldade de marcha, deambulação e equilíbrio.

É possível que variações metodológicas justifiquem as diferentes prevalências encontradas nos estudos. Algumas metodologias estimularam o registro das quedas por meio de calendários, enquanto outros, semelhantes ao presente estudo, analisam a sua ocorrência em uma única entrevista ${ }^{3,9,11}$.

Analisando-se as associações observadas entre queda e intensidade da dor, nota-se, pela tabela 2, que entre os idosos com dor intensa ou moderada relataram maior frequência de quedas do que os idosos com dor leve.

Estudo transversal baseada na Blue Mountains Eye Stu$d y$ (BMES), com indivíduos de 49 anos ou mais, a população de estudo $(n=3509)$ foi examinada pela primeira vez em 1992-94; com reexame pré-agendado para 199799 . Somente $75,1 \%$ da população inicial puderam ser reexaminadas. Os dados foram coletados a partir de entrevistas, exames físicos e questionário contendo perguntas sobre uso de medicamentos e histórico prévio de quedas. Para análise de dor foi utilizado o Bodily Pain Subscale SF-36 ${ }^{1}$. Neste estudo 90,7\% da amostra apresentaram queixa de dor, desses $27,2 \%$ relataram episódios de que- das nos últimos 12 meses e 8,6\% apresentaram episódios de múltiplas quedas. Demonstraram que a dor e a interferência nas atividades de vida diárias (AVD) são mais frequentes em mulheres, $51,4 \%$. As quedas foram mais frequentes em indivíduos com dor moderada e intensa com interferência nas AVD comparadas com os pacientes que não referiram queixas álgicas (prevalência ajustada $1,42 \mathrm{p}=0,0001$ e 1,$62 ; \mathrm{p}=0,00156$, respectivamente) ${ }^{1}$.

Análise realizada com 940 mulheres de 65 anos ou mais, durante um período de 3 anos; apresentando uma somatória de 2,078 quedas para o período, sendo que no primeiro ano e último ano de seguimento $39 \%$ das participantes tiveram pelo menos uma queda, no segundo $36 \%$ das mulheres acompanhadas relataram uma queda. A incidência de quedas foi moderadamente mais elevada entre aquelas com dores musculoesqueléticas generalizadas $(\mathrm{OR}=1,66,95 \%$ com IC $=1,25-2,21$ ), definida como dor nos membros superiores e extremidades inferiores do quadril e nas costas ou tórax com pelo menos escala numérica $>4$ ( moderada $)^{2}$. Um estudo americano comprovou que o uso de analgésico diário foi um fator de proteção para quedas $^{2}$. Os dados obtidos quanto a associação entre queda e dor intensa, observados na presente pesquisa, também são encontrados nas pesquisas de Blyth e col. ${ }^{1}$ e Leveille e col. ${ }^{3}$.

Outro aspecto muito avaliado nos estudos é a influência do local de dor na ocorrência de quedas, apesar de não ter sido encontrado significância estatística nas análises desta pesquisa, cabe a apresentação de alguns achados sobre o tema.

Pesquisa realizada na Tailândia encontrou dor nos pés em 14\% dos idosos (60-80 anos) na proporção de 1:4, entre mulheres e homens, totalizando 213 idosos entrevistados. Os autores citam diferentes causas da dor e encontraram que idosos com dor caminham mais devagar $(1,14 \pm 0,12$ vs. $1,19 \pm 0,12 \mathrm{~m} / \mathrm{s}, \mathrm{p}=0.056) 15$. Quedas foram mais frequentes naqueles com dor, ocorrendo em $29,5 \%$ das mulheres e em $12,9 \%$ dos homens $(\mathrm{p}=$ $0,004)$. As taxas de quedas, nos últimos 6 meses foram mais elevadas em mulheres, principalmente as que apresentavam dor nos pés decorrentes de fasceíte plantar, calos, valgo e metatarsalgia ${ }^{15}$.

Pesquisadores brasileiros ao analisarem 30 indivíduos com mais de 65 anos participantes de um programa de atividades físicas, encontraram que $78 \%$ dos idosos, idade acima de 65 anos, analisados relataram episódios de quedas das quais os ambientes mais citados foram 55\% na rua e $33 \%$ em ambientes fechados (casa, restauran- 
tes, igrejas, outros). Justificando a exposição à queda por idosos mais ativos, estando esses submetidos a fatores ambientais de difícil interferência e que dificilmente são avaliados nas pesquisas de ocorrência de quedas em idosos ${ }^{16}$.

Alguns estudos ${ }^{1-4,10,15}$ avaliaram a ocorrência de queda e alterações na marcha para idosos com dores especificamente localizadas nos membros inferiores (MMII), mas poucos realizaram a comparação com dores em outros locais ${ }^{13}$.

Pesquisadores nos Estados Unidos ao realizarem estudo de coorte com 749 idosos com mais de 70 anos avaliou a poliartralgia e sua relação com quedas. Encontrou a poliartrite como um fator de risco para queda quando comparado com outros locais de dores isoladas como: lombar, quadril, joelho, pés e membros superiores (MMSS). Ao comparar dor dorsal com outros locais de dor, encontrou dores em locais diferentes da região dorsal apresentaram mais risco de queda. O mesmo resultado foi encontrado ao comparar dores no quadril e no joelho com outros locais de dor ${ }^{13}$.

Estudo brasileiro encontrou entre outras variáveis que a ausência de problemas de coluna é um fator proteção para ocorrência de quedas ${ }^{14}$.

Fator que pode ter determinado a não associação entre local de dor e ocorrência de queda nos presentes achados é o fato que se relacionou a análise somente dos locais de dor que mais incomodavam. Assim, idosos com múltiplos locais de dor, foram incluídos somente no grupo da "dor que mais incomoda", mesmo possuindo outros locais de dor, o que pode ter interferido nos resultados.

Outros fatores podem estar associados à ocorrência de quedas em idosos, como os fatores extrínsecos. Um estudo prospectivo observacional com amostra de 765 idosos com 70 anos ou mais, avaliou as condições ambientais como obstáculos no chão, presença de escadas e de equipamentos de adaptação para maior segurança do idoso ${ }^{3}$. No estudo, $39 \%$ dos idosos relataram episódios de queda nos últimos 12 meses anteriores à entrevista inicial e $17 \%$ relataram duas ou mais quedas no mesmo período. Na presente pesquisa fatores ambientais não foram analisados.

Poucas pesquisas exploraram a intensidade e a frequência de dor como fatores de risco para a ocorrência de quedas, o que limitou as comparações com o presente estudo. Porém, as associações observadas demonstraram alguns caminhos a serem seguidos para a prevenção das quedas, como adequada analgesia e orientação sobre sua prevenção.

\section{CONCLUSÃO}

O presente estudo teve como principal resultado a alta prevalência de quedas entre idosos com dor a mais de um ano e a relação entre intensidade da dor e relato de quedas. Os idosos com dor há mais de um ano, de forma intensa ou moderada relataram mais queda do que dos idosos com dores leves. Este tema ainda é controverso na literatura internacional. Não foram encontradas associações entre o local de dor e frequência dos episódios de dor e o relato de quedas no último ano.

\section{REFERÊNCIAS}

1. Blyth FM, Cumming R, Mitchell P, et al. Pain and falls in older people. Eur J Pain 2007;11(5):564-71.

2. Leveille SG, Bean J, Bandeen Roche K, et al. Musculoskeletal pain and risk for falls in older disabled women living in the community. J Am Geriatr Soc 2002;50(4):671-8.

3. Leveille SG, Kiel DP, Jones RN, et al. The MOBILIZE Boston Study: design and methods of a prospective cohort study of novel risk factors for falls in an older population. BMC Geriatr 2008;18(8):16.

4. Lamb SE, McCabe C, Becker C, et al. The optimal sequence and selection of screening test items to predict fall risk in older disabled women: the Women's Health and Aging Study. J Gerontol A Biol Sci Med Sci 2008;63(10):1082-88.

5. Guideline for the prevention of falls in older persons. American Geriatrics Society. British Geriatrics Society, and American Academy of Orthopaedic Surgeons Panel on Falls Prevention. J Am Geriatr Soc 2001;49(5):664-72

6. Dellaroza MS, Pimenta CA, Matsuo T. Prevalence and characterization of chronic pain among the elderly living in the community. Cad Saude Publica 2007;23(5):1151-60.

7. Panazzolo, D, Trelha CS, Dellaroza, MSG., Cabrera MAS, Souza, R.

Dor crônica em idosos moradores do Conjunto Cabo Frio, cidade de Londrina/Pr. Revista Dor 2007;8(3):1047-51.

8. Merskey H, Bogduk N, (editors). Classification of chronic pain: descriptions of chronic pain syndromes and definitions of pain terms prepared by the international association for the study of pain. 2 nd ed. Seatle: IASP Press; 1994. p 227.

9. Barrangán-Berlanga AJ, Mejía-Arango S, Gutiérrez-Robledo LM. Pain in the elderly: prevalence and 
associated factors. Salud Publica Mex 2007;49(Suppl 4):S488-94.

10. Arnold CM, Faulkner RA. The history of falls and the association of the timed up and go test to falls and near-falls in older adults with hip osteoarthritis. BMC Geriatr 2007;4(7):17.

11. Yagci N, Cavlak U, Aslan UB, et al. Relationship between balance performance and musculoskeletal pain in lower body comparison healthy middle aged and older adults. Arch Gerontol Geriatr 2007;45(1):109-19. 12. Bishop MD, Meuleman J, Robinson M, et al. Influence of pain depression on fear of falling, mobility and balance in older male veterans. J Rehabil Res Dev 2007;44(5):675-83.

13. Leveille SG, Jones RN, Kiely DK, et al. Chronic musculoskeletal pain and the occurrence of falls in an older population. JAMA 2009;302(20):2214-21.

14. Perracini MR, Ramos LR. Fall-related factors in a cohort of elderly community residents. Rev Saude Publica 2002;36(6):709-16.

15. Chaiwanichsiri D, Janchai S, Tantisiriwat N. Foot disorders and falls in older persons. Gerontology 2009;55(3):296-302.

16. Guimarães JMN, Farinatti PTV. Análise descritiva de variáveis teoricamente associadas ao risco de quedas em mulheres idosas. Rev Bras Med Esporte 2005;11(5):299-305.

Apresentado em 21 de janeiro de 2011.

Aceito para publicação em 06 de junho de 2011. 